\title{
La función comunicativa de la ponencia. Estructura retórica de un género académico-disciplinar
}

\author{
The communicative function of the paper. Rhetorical structure of an \\ academic-disciplinary genre
}

\author{
Nereyda Álvarez Martínez \\ neret.al@gmail.com \\ Código ORCID: 0000000180286173 \\ Universidad Pedagógica Experimental Libertador, Miranda, Venezuela
}

\section{RESUMEN}

La investigación tuvo como objetivo elaborar una aproximación del género académico ponencia, a fin de identificar en su organización estructural (secciones), los propósitos comunicativos tanto globales (movidas) como locales (pasos). El corpus estuvo conformado por un grupo de ponencias presentadas por escritores expertos en distintos eventos, tanto nacionales como internacionales, cuya área temática se enmarca en la disciplina de la lingüística. El principal resultado fue que se logró caracterizar la estructura retórica de este género. Está organizada en cinco secciones (I. Planteamiento de la investigación, II. Marco teórico y referencial, III. Diseño de la investigación, IV. Resultados y/o Discusión, V. Conclusiones), conformada por siete (7) movidas y trece (13) pasos; además se reveló la presencia reiterativa de la movida retórica citación de autores, como una forma de posicionamiento discursivo frente a una comunidad especializada. La ponencia es un género académico que cumple, entre otras, la función persuasiva.

Palabras clave: Ponencia; secciones; movidas retóricas; pasos retóricos; citación

\section{ABSTRACT}

The objective was to make an approximation of the academic genre, in order to identify in its structural organization (sections), the communicative purposes both global (moved) and local (steps). The corpus was made up of a group of papers presented by expert writers in different events, both national and international, whose thematic area is framed in the discipline of linguistics. The main result was that it was possible to characterize the rhetorical structure of this genre. It is organized into five sections (I. Research approach, II. Theoretical and referential framework, III. Research design, IV. Results and / or Discussion, V. Conclusions), consisting of seven (7) moves and thirteen (13) steps; In addition, the repetitive presence of the cited rhetorical movement of authors was revealed, as a form of discursive positioning in front of a specialized community. In short, the paper is an academic genre that fulfills, among others, the persuasive function.

Keywords: Academic presentation; sections; rhetorical moves; rhetorical steps; quotation 


\section{INTRODUCCIÓN}

La escritura académica constituye una actividad de producción intelectual realizada en contextos sociales específicos vinculados a las disciplinas. Es una forma escritural que demanda el desarrollo de competencias lingüísticas, cognitivas y pragmáticas orientadas a favorecer la tarea de producción escrita de alto nivel. Uno de los ámbitos discursivos donde este tipo de escritura se evidencia es en la investigación, que se materializa en diversos géneros, tales como el artículo de investigación y las tesis, por citar algunos, exigidos en el nivel de educación superior.

Sin embargo, conocer y tener experticia en la producción de textos cuya función es comunicar la investigación no ocurre de forma espontánea. Es una actividad que demanda una acción enfocada a conocer la estructura profunda de géneros académicos con propósitos específicos, a fin de apropiarse de sus esquemas. Es decir, aproximarse al lugar donde residen las intenciones y los propósitos comunicativos. El fin último en todo caso es comprender el discurso disciplinar y desde esas esferas impulsar estrategias pedagógicas. (Parodi, 2008)

Ahora bien, el estudio del discurso especializado se ha abordado desde distintas visiones y métodos, entre las que se destaca el genre analysis, el enfoque del análisis de género (Swales, 1990), una perspectiva de análisis textual que permite comprender cómo se configuran los discursos en atención a sus propósitos comunicativos. Su propuesta surge a partir del análisis de las introducciones del artículo de investigación en el idioma inglés, a la que se fueron sumando estudios orientados a otras secciones y géneros empleados para comunicar la investigación.

Se trata de una perspectiva que se fue enriqueciendo y ampliando a otros idiomas como el español, convirtiéndose en una opción de abordaje para reconocer e identificar la estructura retórica que subyace en distintos géneros académicos y profesionales.

El presente estudio tuvo como objetivo aproximarse a un tipo de comunicación cuya función es la difusión de la investigación en ámbitos socios discursivos especializados. Se trata de la ponencia; escasamente estudiada como género académico, pero siempre requerida en contextos vinculados a encuentros de investigación de distinta naturaleza y disciplina. La investigación se puso en marcha con el fin de abordar la construcción textual y discursiva de este género, bajo el enfoque de movidas y pasos que es la base de la propuesta de Swales (1990) y que Sabaj, Toro y Fuentes (2011) operacionalizaron en español.

La identificación de la estructura retórica de la ponencia representa un aporte pedagógico que se reflejará en la enseñanza de la escritura de este género 
de exigencia frecuente en educación superior, con énfasis en el postgrado.

El ejercicio de la lectura y la escritura en la universidad debe hacerse desde las disciplinas, con el fin de que los aprendices accedan a conocimientos culturales específicos que les permitan producir ciertos tipos de géneros académicos con el fin de presentar a través de ellos su hacer investigativo. Por ello, es un desafío para los estudiantes apropiarse de los razonamientos y convenciones que caracterizan a la comunidad discursiva a la que se vinculan y a los géneros que circulan en ella, pues cada género obedece a particularidades específicas para comunicar las experiencias académicas. (Carlino, 2009)

En cuanto a la Educación Superior, la expectativa es que los estudiantes se incorporen a la cultura escrita de las disciplinas y esto acarrea aproximarse a conceptos y patrones comunicativos específicos. En estos espacios la escritura rebasa los límites de una cátedra o de una disciplina, por ello es necesario incorporarla en cada área curricular. (Carlino, 2005)

Es preciso entender que a medida que se escribe se transforma el pensamiento del escritor en relación al tema que ha estado desarrollando, es decir, que hay un poder epistémico asociado a la escritura. En cuanto a los escritores expertos, este valor epistémico de la escritura les permite no solo ir adquiriendo cada vez más claridad acerca de su producción, sino poner en sintonía el conocimiento con los propósitos comunicativos y el contexto de recepción. Mientras que los escritores menos experimentados tienen una visión más limitada y unidireccional de su texto (Scardamalia y Bereiter, 1985). Es por ello que algunas universidades se han alineado con la corriente denominada escribir desde el currículo, que otros han llamado escribir desde las disciplinas,

En la investigación científica, por ejemplo, existe una suerte de prototipos o superestructuras textuales que deben seguirse para hacer el reporte investigativo. Tal como ocurre en la producción escrita de géneros como la tesis o el artículo de investigación, en los que los autores deben apegarse a pautas muy prescriptivas. El escritor debe elaborar su texto en función de la estructura convencional (introducción, planteamiento del problema, marco teórico, resultados, análisis de los resultados y conclusiones), para registrar los distintos momentos del proceso de investigación. No solo se trata de apropiarse de aspectos estructurales, sino de incorporarse $y$ aprehender las convenciones propias de la disciplina, es decir, introducirse en una cultura discursiva específica.

En los estudios superiores hay géneros académicos que típicamente son exigidos (tesis, artículos de investigación, informes, las ponencias, 
entre otros) y cada uno responde a una forma de organización textual. Esa estructura prototípica característica de cada género discursivo obedece así mismo a una estructura interna, que corresponde a su organización retórica, misma que da cuenta de los propósitos comunicativos que caracterizan la investigación dentro de su comunidad discursiva.

Ahora bien, dentro del amplio espectro de géneros académicos se encuentra la ponencia, un tipo de texto de exigencia frecuente en el postgrado para comunicar la investigación y validar resultados. Empleada por escritores nóveles y expertos, es un género que de acuerdo a su función contextual y discursiva contribuye a posicionar a los investigadores dentro de una determinada comunidad de expertos. Constituye una producción cuya función es la comunicación del conocimiento permitiendo al autor construir su propia voz como investigador en espacios discursivos especializados.

En este artículo se muestran los rasgos textuales y discursivos de la ponencia, para reconocer cómo se conforma su función comunicativa (estructura retórica), de acuerdo con la perspectiva del análisis de géneros (Swales, 1990).

La ponencia es un tipo de texto de obligatoria exigencia para presentar investigaciones en el marco de convocatorias a eventos especializados nacionales y foráneos, participación que también es exigida como requisito de egreso en algunos doctorados del país. Sin embargo, no ha sido objeto de investigación como género académico en cuanto a la estructura retórica que orienta su construcción. De modo que es necesario aproximarse a las ponencias con el fin de determinar sus propósitos comunicativos y organizarlos en términos de las movidas y pasos que la conforman.

El propósito de la investigación fue aproximarse hacia la construcción de un modelo prototípico que dé cuenta de la organización retórica del género seleccionado.

El resultado pretende ser un aporte que contribuya al desarrollo de la escritura de géneros académicos en los niveles de pre y postgrado del Instituto Pedagógico de Miranda "José Manuel Siso Martínez", adscrito a la Universidad Pedagógica Experimental Libertador.

\section{Objetivo general}

Analizar la organización retórica de la ponencia como género de difusión de la investigación.

\section{Objetivos específicos}

- Identificar los propósitos comunicativos globales (Movidas retóricas) y los propósitos locales (pasos) 
- Describir los propósitos comunicativos globales (Movidas retóricas) y los propósitos locales (pasos)

- Identificar la estructura prototípica que subyace al género ponencia desde el enfoque del análisis de género

Estudios orientados a la identificación de la estructura retórica, como el planteado aquí, son escasos a nivel de la UPEL, no encontrándose trabajos previos en el Instituto Pedagógico de Miranda. Otro aspecto que revaloriza esta aproximación investigativa es su aporte al conocimiento de la escritura académica en el área de lengua. Dado el rol que los docentes desempeñan en la formación de escritores competentes, quienes deben ajustar su forma de leer y escribir, en función de la comunidad en la que participan (Carlino, 2005). De manera que producir géneros académicos requiere del dominio de ciertas habilidades por parte de los miembros de una comunidad y por ello es fundamental conocer la estructura retórica convencional de los mismos (Kanotsilaphatan, 2005, citado en Morales 2010).

El enfoque asumido en esta investigación corresponde al de movidas y pasos retóricos propuesto originalmente por Swales (1990), el cual propone la fragmentación de un texto en segmentos con el fin de identificar los propósitos comunicativos que subyacen a los mismos. Es decir, cada una de las movidas en que se segmenta un texto constituye una sección que revela una función comunicativa específica y aporta tanto el propósito comunicativo global del género como la forma específica que lo distingue de otros. Esta organización global es guiada por los propósitos comunicativos subyacentes, que se articulan progresivamente en un todo que se realiza de forma lingüística. Es esta estructura subyacente e incrustada en la superficie textual la que el investigador debe hacer visible a través de la identificación de las movidas y los pasos retóricos (Parodi, 2010).

El énfasis de este enfoque recae en la organización retórica de los textos producidos en contextos académicos y científicos, como una forma de alfabetizar tanto académica como científicamente. En esta misma línea, se inscribe el análisis de Artículos de Investigación en español que se propuso describir las distintas etapas presentes en la construcción de un modelo de movidas retóricas (Sabaj, Toro y Fuentes, 2011). Para estos autores la Movida Retórica se define como "la expresión de un propósito comunicativo que se asocia a un fragmento textual y que contribuye al logro del propósito global de un género" (p.247). Mientras que otra de las definiciones señala que " $A$ 'move' in genre analysis is a dicoursal and rhetorical unit performs a coherent communicative function in a written or spoken discourse" "'Un 'movimiento' en el análisis de género es una unidad 
discursiva y retórica que realiza una función comunicativa coherente en un discurso escrito o hablado" (Swales, 1990).

Los estudios orientados con el enfoque de movidas y pasos de textos o segmentos de textos académicos en español, se derivan de los primeros estudios que estuvieron enfocados hacia el estudio del Inglés con Propósitos Específicos (ESP), vinculado al campo de la enseñanza de la escritura científica de esta lengua. Los estudios pioneros en esta línea de investigación se concentraron en indagar las MR en secciones puntuales como las introducciones.

\section{El modelo Create a Research Space (CARS)}

Esta orientación comprende el análisis del uso del lenguaje en un sentido más amplio, que toma en cuenta no solo la forma como se construye el texto, sino cómo este puede ser interpretado $\mathrm{y}$ empleado con fines específicos a fin de lograr objetivos también específicos.

En el campo de la lingüística aplicada, es un análisis que se utiliza en la investigación con el fin de describir la estructura y características estilísticas de los textos. La propuesta en cuestión apunta hacia la construcción de un modelo de movidas retóricas para el análisis de artículos de investigación en español. Al igual que, en el trabajo que nos ocupa uno de los objetivos busca determinar la estructura retórica que subyace al género académico ponencia, que luego podría servir como referencia para la construcción de este tipo de texto. Las MR y los pasos contribuyen a expresar los propósitos comunicativos en niveles distintos.

El proceso para la construcción de un modelo de MR de acuerdo a lo señalado por Sabaj et al. (2011), consta de varias operaciones cognitivas de análisis textual y síntesis de la información que de forma general puede especificarse de la siguiente manera: a) Indagación sistemática del género seleccionado, $y \quad b)$ Homologación de la información $\mathrm{y}$ construcción del modelo. En ambas etapas se utilizan procedimientos diferenciados como: técnicas de registro de información, análisis textual y homologación.

\section{Géneros académicos}

De acuerdo a lo que señala Swales (1990), (citado en Venegas, Meza y Martínez, 2013) "el género es un evento comunicativo que tiene propósitos identificados y definidos por los miembros de una comunidad académica o profesional determinada, quienes construyen sus géneros a partir de formas o contenidos particulares" (p.156)

Otra descripción de género discursivo es la que ofrece Parodi (2009, 2010), a partir de su enfoque socio 
cognitivista de los géneros discursivos con énfasis en su manifestación escrita. Tal enfoque, como él mismo señala, dista mucho de estar acabado, sin embargo, se apoya en los principios ontológicos y epistemológicos de la concepción del ser humano y del lenguaje, en consecuencia toca la concepción integral y multidimensional de los géneros, fundamentos esenciales, según señala, para explorar a teoría de los géneros académicos y profesionales así como para promover comprender o dilucidar la alfabetización disciplinar especializada.

$\mathrm{El}$ género viene a ser un constructo que permite reunir textos que comparten ciertos rasgos, no solo de tipo contextual (propósitos comunicativos), sino lingüísticos y discursivos, entre los que se figura la estructura retórica y la organización discursiva. Cuando el propósito comunicativo es uno de los rasgos que predominantemente comparten, se establece una regularidad $\mathrm{y}$ en consecuencia pueden ser identificados como pertenecientes a un género. (Swales, 1990)

\section{La ponencia}

Para Duo de Brottier (2007) “...la ponencia es la realización de un acto comunicativo que se lleva a cabo en reuniones de especialistas y su finalidad es proporcionar conocimiento o dar lugar a la discusión" (p.113). Briz (2008) citado en Villar (2011), consideran que la ponencia es una variante de la conferencia.

La limitada información orientada hacia la construcción retórica de este género hace pensar que ha sido poco estudiada, a pesar de ser un tipo de comunicación de exigencia obligada cuando se trata de comunicar la investigación.

Es utilizada para comunicar resultados de investigaciones concluidas o en proceso, lo que le confiere una macrofunción especifica como texto de difusión. Según lo que reporta Cubo de Severino (2005) se deriva de una investigación y por lo tanto está dotada de una estructura de base sobre la cual se organiza la información. De manera que la ponencia responde a la noción de género según la perspectiva de Swales (1990), quien equipara género con un evento comunicativo cuyo propósito está bien definido de acuerdo a las convenciones asumidas por una comunidad académica y profesional específica.

Desde el punto de vista contextual la presentación de ponencias contribuye a posicionar las investigaciones y la presencia autoral dentro de un ámbito académico específico para difundir $\mathrm{y}$ promover enfoques, perspectivas metodológicas $\mathrm{u}$ otros aspectos relevantes vinculados con la investigación. Constituyen presentaciones alineadas a una temática disciplinar específica que se 
presentan en un espacio y tiempo determinado. En consecuencia, la ponencia puede ser incluida y definida como un género, pues goza de una serie de rasgos que le atribuyen una tipología particular y por ende, responde a una configuración retórica en la que subyacen propósitos comunicativos específicos.

\section{MÉTODO}

La investigación se encuadra en el seno de prácticas socio discursivas cuya finalidad es reconocer los propósitos comunicativos que subyacen al género académico ponencia. Se trata de comprender e interpretar cómo se comunica la investigación a partir de textos escritos que fueron objeto de revisión preliminar en el marco de eventos reales. La identificación de los propósitos comunicativos, como ya se ha señalado estará guiada por la perspectiva teórico-metodológica del análisis de género. (Swales, 1990; Sabaj, Toro y Fuentes, 2011)

La perspectiva teórica asumida responde al enfoque hermenéutico ya que se espera dar cumplimiento a los objetivos atendiendo a la interpretación del objeto investigado, (hermenéutica significa interpretar o comprender). Con el concurso de los lineamientos aportados por el análisis de género que busca identificar las movidas y pasos (propósitos comunicativos globales $\mathrm{y}$ locales) (Sabaj, Toro y Fuentes, 2011), el cual está inmerso dentro del método de análisis del discurso.
La técnica empleada para la recolección de la información fue la revisión documental, que consistió en explorar en diversas fuentes consideradas confiables. Los textos recopilados fueron obtenidos partir de la búsqueda en actas de congresos de renombre a nivel nacional e internacional publicadas en la web. La información y aspectos vinculados al corpus seleccionado se organizó y codificó para facilitar el posterior análisis. (ver Tabla 1)

Con el objeto de definir el corpus se cita a Santalla del Río (2005) quien señala que:

...es un conjunto de textos de lenguaje natural e irrestricto, almacenados en un formato electrónico homogéneo, $\mathrm{y}$ seleccionados y ordenados, de acuerdo con criterios explícitos, para ser utilizados como modelo de un estado o nivel de lengua determinado, en estudios $\mathrm{O}$ aplicaciones relacionados en mayor o menor medida con el análisis lingüístico. (pp. 45-46)

Para realizar la investigación se compiló un corpus de seis (6) ponencias presentadas por escritores expertos nacionales $\mathrm{y} / \mathrm{o}$ internacionales de reconocida trayectoria académica, con el propósito de garantizar que los resultados se fundamenten sobre la base del análisis de producciones de escritores competentes. Otro de los rasgos que unifican el corpus seleccionado es el área disciplinar en las que se inserta cada 
producción, que en este caso fue la lingüística. Los rasgos considerados para la recolección del corpus responden a varios criterios, entre los que se señalan: a) Escritores expertos, b) Presentados en eventos nacionales y/o internacionales, c) Autores adscritos a instituciones universitarias, d) Textos enmarcados en el área lingüística.

\section{Procedimiento:}

- Segmentación de los textos de las ponencias en párrafos

- Identificación de las secciones o macromovidas de cada ponencia, las macromovidas vienen a conformar lo que sería la superestructura que sostiene todo el cuerpo textual y contiene las unidades comunicativas globales y locales (movidas y pasos)

- Identificación de propósitos comunicativos locales o pasos, a partir de la interpretación del contenido del texto.
- Generación de las movidas retóricas en función de la articulación de distintos pasos.

- Homologación de la información: este procedimiento se realizó a partir de los siguientes subprocesos: D.1síntesis consiste en fusionar en uno solo los pasos o movidas retóricas redundantes D.2-modificación se elimina la ambigüedad de alguna categoría. D3-adición la suma de propósitos comunicativos no contemplados, pero que pudieran emerger del procesamiento de los datos. Este modelo de procesamiento de la información se deriva de los insumos que provee la propuesta de Sabaj et al. (2011)

Aproximación un modelo prototípico característico de las ponencias en el área de lingüística a partir de la organización de las secciones, movidas retóricas y pasos. 
Tabla 1. Descripción del corpus de estudio. Tipo de discurso: ponencias. Área: lingüística

\begin{tabular}{|c|c|c|c|c|}
\hline TÍTULO & AUTOR & $\begin{array}{l}\text { UNIVERSIDAD DE } \\
\text { PROCEDENCIA }\end{array}$ & EVENTO/FECHA & CÓDIGO \\
\hline $\begin{array}{l}\text { 1. Discurso escrito, relaciones } \\
\text { de poder y comunidades del } \\
\text { conocimiento }\end{array}$ & $\begin{array}{l}\text { Rudy Mostacero } \\
\text { RM1 }\end{array}$ & $\begin{array}{l}\text { Universidad Pedagógica } \\
\text { Experimental Libertador. } \\
\text { Instituto Pedagógico de Maturín }\end{array}$ & $\begin{array}{l}\text { Foro sobre el Día del Idioma } \\
\text { (IPMAR) } \\
\text { (2012) }\end{array}$ & PUPELMAT12-1 \\
\hline $\begin{array}{l}\text { 2. Empleo de metáforas } \\
\text { conceptuales con fines } \\
\text { argumentativos en el discurso } \\
\text { político venezolano }\end{array}$ & Thays Adrián TA2 & $\begin{array}{l}\text { Universidad Pedagógica } \\
\text { Experimental Libertador. } \\
\text { Instituto Pedagógico de Caracas }\end{array}$ & $\begin{array}{l}\text { II Conferencia Internacional: } \\
\text { Lógica, argumentación y } \\
\text { pensamiento crítico (2011) }\end{array}$ & PUPELIPC11-2 \\
\hline $\begin{array}{l}\text { 3. Las huellas del proceso de } \\
\text { producción de un documento: } \\
\text { tratado constitutivo de la } \\
\text { unión de naciones } \\
\text { suramericanas (UNASUR) }\end{array}$ & $\begin{array}{l}\text { Elvira Arnoux y } \\
\text { Florencia Magnanego } \\
\text { AYM3 }\end{array}$ & $\begin{array}{l}\text { Universidad Nacional de } \\
\text { General Sarmiento, Argentina }\end{array}$ & $\begin{array}{l}\text { Congreso Regional de la Cátedra } \\
\text { UNESCO en Lectura y Escritura. } \\
\text { Cultura Escrita y Políticas } \\
\text { Pedagógicas en las Sociedades } \\
\text { Latinoamericanas Actuales. (2010) }\end{array}$ & PUNGS10-3 \\
\hline $\begin{array}{l}\text { 4. La comunicación académica } \\
\text { como construcción } \\
\text { argumentativa: perspectivas } \\
\text { de lectura y de escritura } \\
\text { de estudiantes universitarios }\end{array}$ & $\begin{array}{l}\text { Silvina Douglas, Esther } \\
\text { López, Constanza } \\
\text { Padilla DLYP4 }\end{array}$ & $\begin{array}{l}\text { Universidad Nacional de } \\
\text { Tucumán. Buenos Aires, } \\
\text { Argentina }\end{array}$ & $\begin{array}{l}\text { XII Congreso de la Sociedad } \\
\text { Argentina de Lingüística (SAL) } \\
\text { (2010) }\end{array}$ & PUNT11-4 \\
\hline $\begin{array}{l}\text { 5. Estrategias retóricas } \\
\text { argumentativas y campaña } \\
\text { electoral } 2006\end{array}$ & Yoselli Briceño B5 & $\begin{array}{l}\text { Universidad Central de } \\
\text { Venezuela (Caracas) }\end{array}$ & $\begin{array}{l}\text { XXVI Encuentro nacional de } \\
\text { Docentes e Investigadores de la } \\
\text { lingüística UPEL-Maracay (2007) }\end{array}$ & PUCV07-7 \\
\hline $\begin{array}{l}\text { 6. "El pueblo" en las elecciones } \\
\text { presidenciales: análisis crítico } \\
\text { de los anuncios de campaña } \\
\text { de Chávez y Rosales }\end{array}$ & $\begin{array}{l}\text { Vanessa Courleander } \\
\text { VC6 }\end{array}$ & $\begin{array}{l}\text { Universidad Metropolitana } \\
\text { UNIMET (Caracas) }\end{array}$ & $\begin{array}{l}\text { XXVI Encuentro nacional de } \\
\text { Docentes e Investigadores de la } \\
\text { lingüística UPEL-Maracay (2007) }\end{array}$ & PUNIMET07-8 \\
\hline
\end{tabular}




\section{RESULTADOS Y DISCUSIÓN}

En cuanto a las secciones, en la revisión realizada se constató que, al menos en el caso de las ponencias del área de lingüística, los contenidos se organizaron en secciones claramente identificables susceptibles de ser segmentadas y caracterizadas según su función comunicativa. De acuerdo con esto, se determinó que la estructura general de las ponencias se organizaría en torno a cinco secciones a saber: I. Planteamiento de la investigación, II. Marco teórico y referencial, III. Diseño de la investigación, IV. Resultados y/o Discusión y V. Conclusiones. Cada una de estas secciones está conformada por movidas retóricas que se integran y portan los propósitos comunicativos globales que caracterizan a este género.

\section{Estructura retórica de las ponencias del área de lingüística (ER-PAL)}

Los resultados de la investigación permitieron reconocer las movidas y pasos que conforman el género seleccionado. Las movidas (MR) identificadas encuentran su realización en una serie de pasos $(\mathrm{P})$ que van mostrando los propósitos locales de acuerdo a lo que el autor desea comunicar, estos pasos no necesariamente van a responder a un orden fijo, sino que van a estar repartidas en distintas posiciones de la movida de acuerdo a las decisiones comunicativas que determine el autor del texto. A continuación, se muestran cada una de las secciones, movidas y pasos que conforman la estructura general de la ponencia.

\section{Planteamiento de la investigación}

En esta primera sección se posiciona discursivamente la investigación al establecer el contexto al cual se circunscribe (MR1 contextualización de la investigación). Presenta una serie de aspectos orientados a configurar el espacio de investigación, el contexto, tanto histórico como legal, educativo, social y discursivo (de acuerdo al interés de investigación). Así mismo, comienza a dilucidarse la temática (P1) y se ofrecen los argumentos necesario para justificar la investigación que se presenta (P2), otro tanto ocurre con el objeto de investigación delineado en el marco al cual se circunscribe y aquel dentro del cual se contextualiza el estudio (P3). A continuación, un ejemplo de lo señalado:

1- a)- Los profesionales que trabajan en las comunidades científicas se caracterizan por sostener interacciones relacionadas con la producción y transformación del conocimiento. En dichas comunidades se intercambian prácticas, saberes y competencias que pertenecen a disciplinas o áreas muy específicas del saber. La especificidad se asocia con los fines y propósitos de cada área, con el uso del lenguaje, pero sobre todo, con la producción particular de géneros y textos que en cada caso dependen de normas y 
tradiciones. Por eso, el uso del discurso identifica $y$, a la vez, diferencia a unos investigadores de otros: novatos y expertos. ( RM1)

b) El discurso político se erige en el lugar donde se lleva a cabo la lucha por el poder. El poder moderno, discursivo (Van Dijk, 2009), cuestiona o consolida el orden existente. Entre los objetivos del análisis crítico del discurso está el de propiciar conciencia discursiva en el entendido de que esta nos conduce al camino de la emancipación, además, favorece la tolerancia, la integración dentro del entorno social $\mathrm{y}$ enriquece el debate público (Martín y Whittaker, 1998). (TA2)

Se comienzan a esbozar los fundamentos teóricos de la investigación (P4), así como los metodológicos (P5), ya en este apartado quedan expuestos los objetivos, preguntas e hipótesis de la investigación (P6) como puede verse en los siguientes ejemplos:

2- a) En cuanto a la discusión de estos datos, los compararemos con resultados obtenidos por investigaciones francófonas que abordan la escritura académica como construcción de saberes (Delcambre y Reuter, 2002; Pollet, 2004), y con resultados de estudios anglófonos que consideran los modos de argumentación en papers de los estudiantes, a partir del trabajo con datos empíricos
(Kelly y Bazerman, 2003).
(DLYP4)

b) Me he planteado las siguientes preguntas para el estudio: ¿cuáles estrategias retóricas argumentativas aparecen en los avisos de campaña y/o de gestión de los candidatos presidenciales Rosales y Chávez? y ¿qué funciones cumplen tales estrategias? (B5)

\section{Marco teórico y referencial}

En esta sección comienzan a exponerse consistentemente los fundamentos teóricos (P7), así como la valoración de la investigación, bien por medio de la citación de los referentes, antecedentes o de otros autores, También hay espacio para hacer una valoración personal de la investigación (P8), observe los siguientes ejemplos:

3- a) Desde finales del siglo XX, Lakoff y Johnson (1980) investigan el rol de la metáfora en el lenguaje habitual; sostienen que esta resulta esencial a la comprensión humana y que el sistema conceptual del hombre está integrado por metáforas básicas que se proyectan en el sistema lingüístico en forma de expresiones metafóricas. (TA2)

b) Según Bolívar (2001), los insultos en el discurso político venezolano son formas de descalificar al oponente, puesto que afectan la 
imagen de los interlocutores con el propósito de reforzar la propia imagen del enunciador, ganar seguidores y llegar o mantenerse en el poder. Por eso, el efecto perlocutivo del uso del insulto en nuestra sociedad es tan eficiente $\mathrm{y}$ poderoso, dadas las reacciones que produce en el común de la población. $\mathrm{El}$ insulto entre los venezolanos ha funcionado como estrategia política que implica la afiliación y la distinción entre grupos y permite mantener el statu quo establecido desde hace unos años hasta nuestros días. (VC6)

\section{Diseño de la investigación}

En esta sección se reúnen los aspectos que conforman el cuerpo metodológico que guio la investigación. Para ello las MR5 se despliega en una serie pasos que esbozan los referentes teóricos que guiarán la investigación (P4) y se delimitan los objetivos (P6) entre los aspectos más generales, como se ejemplifica:

4- a) Para llevar a cabo esta investigación se han seguido algunos de los lineamientos propuestos por Reisigl (2008), particularmente en lo que respecta a la selección y análisis de la data. (TA2)

b) Como recién señalamos, nuestro objetivo es observar si los estudiantes logran capitalizar el conocimiento acerca de los modos de organización del discurso académico-científico, específicamente, de sus partes $\mathrm{y}$ segmentos textuales. Para ello, tanto en la instancia de intervención didáctica como para el análisis de los datos de investigación, seguimos parcialmente los aportes de Swales (1990),Paltridge (1997), Gnutzmann y Oldenburg (1991) y Ciapuscio y Otañi (2002), entre otros, en cuanto a la organización del artículo científico y los aportes de Cubo de Severino (2000) y Dúo de Brottier(2005). (DLYP4)

Por otra parte, al igual que los artículos de investigación, tiene como fin principal mostrar el modelo metodológico que guía la investigación (MR5 Descripción del modelo metodológico de la investigación); de manera que puede encontrase la descripción del corpus (P9), los procedimientos (P10) y las categorías de análisis. (P11)

5- a) El corpus completo de nuestra investigación está integrado por diversas fuentes de datos obtenidos, a lo largo de los cinco años de esta experiencia de investigación-acción: ponencias en sus distintas versiones, testimonios metadiscursivos de los estudiantes, comentarios de los tutores, fichado de material bibliográfico, exámenes parciales, entre otros tutores, fichado de material bibliográfico, exámenes parciales, entre otros (DLYP4) 
b) Categorías retóricas argumentativas: Las estrategias argumentativas utilizadas para el análisis se fundamentan en la clasificación de Perelman y OlbrechtsTyteca señalada por Cross (2003). Entre ellas tenemos: Las estrategias que apelan a la RAZÓN y Las basadas en el argumento de AUTORIDAD. (B5)

c) Como unidad de análisis se tomaron los artículos de noticia que reseñaron los eventos comprendidos por el momento en que se produce el insulto, así como la intervención del presidente en el acto de juramentación de su gabinete. Como categorías de análisis se siguieron las propuestas por Bolívar (2001) las cuales contemplan: el momento del evento, los actores, la reacción verbal de la audiencia y los efectos sociales que se derivaron de ella. (VC6)

\section{Resultados y /o discusión}

Esta sección se sustenta en dos movidas la MR6 (Comunicación de resultados) que se evidencia cuando el autor da cuenta de la investigación justificándola y mencionando la importancia de la investigación (P2), retoma los fundamentos metodológicos (P5) y trae a colación los resultados valorándolos $\mathrm{y} / \mathrm{o}$ contrastándolos. (P8)
6- a) El insulto del presidente Chávez al secretario general de la OEA podría interpretarse de diversas formas, pero sin lugar a dudas ha sido comprendido y catalogado por un sector de la población como un acto descortés. Este uso de la palabra y de su posición como líder máximo de la nación podría explicarse en términos de los eventos que lo generaron. La transformación social que ha implicado el proyecto político del mandatario, ha producido resistencia por parte de la comunidad nacional e internacional, y la suspensión de un canal de TV pareciera indicar la profundidad de los cambios a producirse en el país. Por otro lado, y como ya ha sido estudiado (Bolívar, 2001, 2003, 2005), el carácter confrontacional de esta administración ha definido muchas de las acciones ejecutadas a lo largo de los períodos de gobierno que Chávez ha dirigido, por lo que se ha catalogado como un estilo propio que es capaz de transgredir las fronteras nacionales. (VC6)

b) Esta muestra resulta suficiente para observar que en las alocuciones del expresidente Rómulo Betancourt dichas figuras constituyen un relevante organizador argumental, en virtud de que su comprensión exige un mínimo esfuerzo de procesamiento. Se trata de tropos ya lexicalizados con fuertes implicaturas. (TA2) 
La segunda movida identificada en esta sección MR2 (citación de autores) describir e interpretar los resultados convocando voces autorizadas.

7- a) Como lo afirma Bolívar (2001, 2003, 2005) la descortesía se ha materializado en nuestro país en diversas formas, una de ellas son los insultos. Este tipo de actos no contribuyen con el equilibrio de la interacción $\mathrm{y}$ ha degenerado en violencia física y verbal. De esta manera, estas prácticas discursivas y sociales se han ido naturalizando en nuestro país. (VC6)

b) Las investigaciones francófonas, en el análisis de las relaciones entre escritura y construcción de conocimiento, postulan representaciones estudiantiles que oscilan entre la concepción de la escritura como transcription de la pensée versus construction de la pensée (Delcambre y Reuter, 2002), o comostockage -almacenamientoversus élaboration (Pollet, 2004), atendiendo a indicadores tales como el trabajo con las fuentes bibliográficas y la distancia enunciativa. (DLYP4)

\section{Conclusiones}

Se muestran las conclusiones confirmando la justificación y el objetivo de investigación, se valoran los resultados (MR7 Formulación de las conclusiones). Se retoma y justifica la investigación (P2), el propósito es persuadir al despertar el interés o adhesión bien hacia la investigación como tal o hacia algún aspecto puntual de esta; el objeto de investigación, la temática, la metodología empleada, los resultados y /o la continuidad de la misma (P8). Es el espacio para presentar y discutir los resultados (P13). Se observa que persiste la citación de autores con la función específica de respaldar y apoyar los resultados bien para recomendar, criticar, comentar o aclarar, lo que sugiere el posicionamiento del trabajo presentado ante la audiencia (MR2 Citación de autores).

8- a) Y para el caso del nivel superior, se añade un factor más, junto con la desatención y la falta de responsabilidad, por parte de las autoridades universitarias $\mathrm{o}$ ministeriales, se constata el uso del discurso académico para incrementar y mantener las diferencias de estatus, vale decir, de poder, porque como lo dice Charaudeau (2004), a los investigadores no sólo se les reconoce por su formación $\mathrm{y}$ por sus publicaciones, sino porque utilizan su estatus académico para imponer algún tipo de poder. (RM1)

b) Los dilemas de la integración en el proceso de construcción de un imaginario colectivo solo son resueltos a través de la escritura que pone en orden y sutura las grietas transitoriamente a través de 
definiciones imprecisas que yuxtaponen conceptos conflictivos, olvidos y elisiones, expresiones elípticas plagadas de subordinantes y adjetivos que convocan nuevos sentidos, fórmulas consolidadas $\mathrm{y}$ lugares comunes. El consenso, abierto a la transformación y la controversia, se estabiliza en la producción del documento. La escritura resuelve pero no anula las múltiples tensiones que atraviesan el proceso de integración suramericana. (AYM3)

\section{Construcción del modelo prototípico}

Una vez realizado el proceso de homologación se construyó el modelo prototípico (ver Tabla 2) a partir de las movidas y pasos resultantes. Se recopiló también el número de movidas y pasos que se determinaron por sección (ver Tabla 3). El análisis realizado refleja la presencia de pasos en forma recurrente, como en el caso de la citación de autores, da señales de su importancia desde el punto de vista del propósito comunicativo, de manera que se constituye en una movida que se muestra en cuatro de las cinco secciones identificadas, lo cual es un rasgo que se puede presentar al momento de construir la organización retórica de cualquier género. (Sabaj et al., 2011)

Tabla 2. Estructura retórica identificada para el género académico ponencia

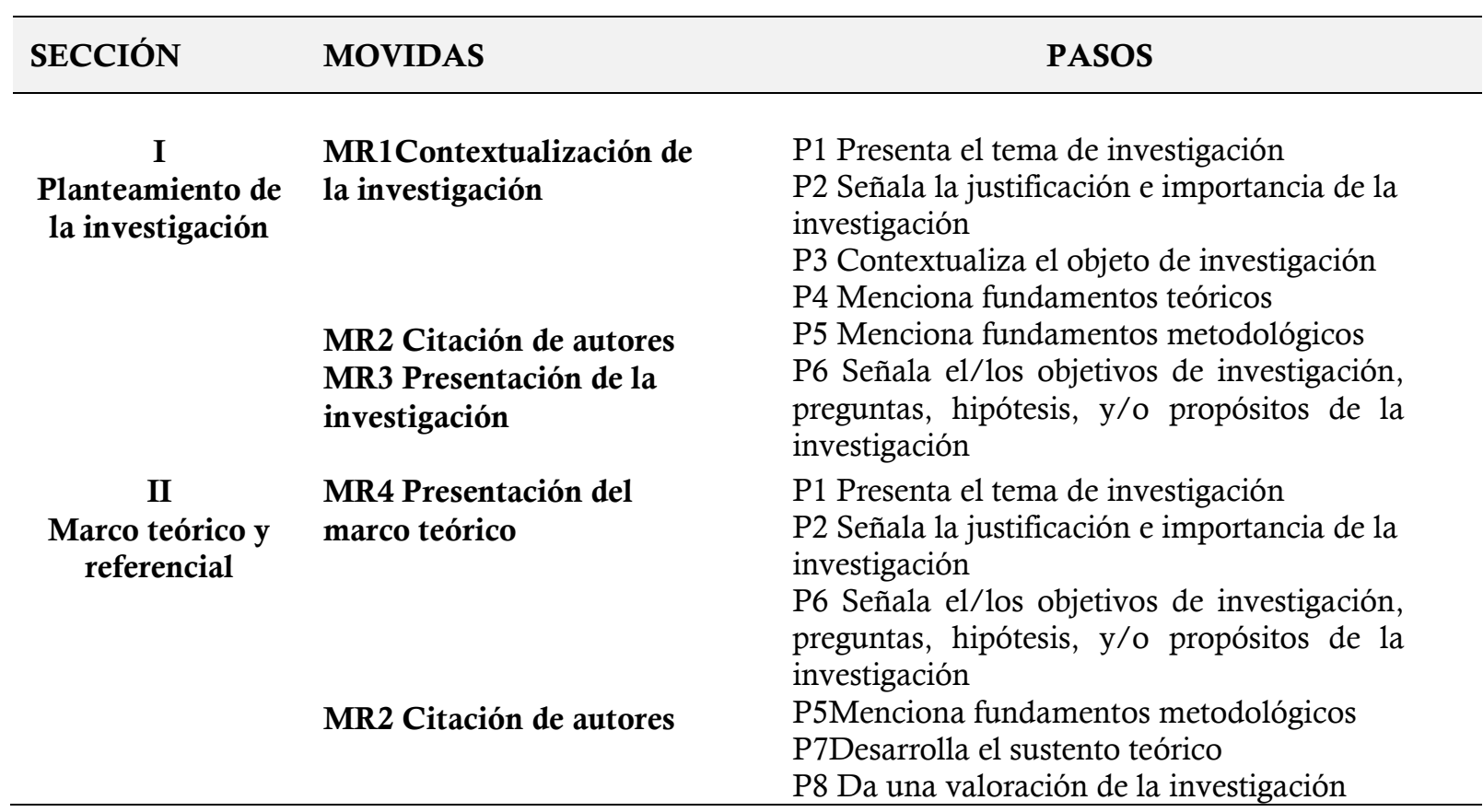




\begin{tabular}{|c|c|c|}
\hline SECCIÓN & MOVIDAS & PASOS \\
\hline $\begin{array}{c}\text { III } \\
\text { Diseño de la } \\
\text { investigación }\end{array}$ & $\begin{array}{l}\text { MR5 Descripción del } \\
\text { modelo metodológico de la } \\
\text { investigación }\end{array}$ & $\begin{array}{l}\text { P6 Señala el/los objetivos de investigación, } \\
\text { preguntas, hipótesis, y/o propósitos de la } \\
\text { investigación } \\
\text { P4 Menciona fundamentos teóricos } \\
\text { P9 Describe el corpus } \\
\text { P10 Describe los procedimientos } \\
\text { P11 Describe las categorías de análisis }\end{array}$ \\
\hline $\begin{array}{l}\text { IV } \\
\text { Resultados y/o } \\
\text { discusión }\end{array}$ & $\begin{array}{l}\text { MR6 Comunicación de } \\
\text { resultados }\end{array}$ & $\begin{array}{l}\text { P2 Señala la justificación e importancia de la } \\
\text { investigación } \\
\text { P5 Menciona fundamentos metodológicos } \\
\text { P8 Da una valoración de la investigación } \\
\text { P12Explica describe e interpreta resultados }\end{array}$ \\
\hline $\begin{array}{c}\mathrm{V} \\
\text { Conclusiones }\end{array}$ & $\begin{array}{l}\text { MR7 Formulación de las } \\
\text { conclusiones }\end{array}$ & $\begin{array}{l}\text { P2 Señala la justificación e importancia de la } \\
\text { investigación } \\
\text { P13 Discute los resultados } \\
\text { P5Menciona fundamentos metodológicos } \\
\text { P8 Da una valoración de la investigación }\end{array}$ \\
\hline
\end{tabular}

Tabla 3. Registro de movidas y pasos identificadas por sección

\begin{tabular}{lcc}
\hline SECCIÓN & NÚMERO DE PASOS & NÚMERO DE MOVIDAS \\
\hline I. Planteamiento de la investigación & 6 & 3 \\
II. Marco teórico y referencial & 6 & 2 \\
III. Diseño de la investigación & 5 & 1 \\
IV. Resultados y/o Discusión & 4 & 2 \\
\hline
\end{tabular}

Algunos de los hallazgos resultantes muestran que hay un fenómeno de repetición de movidas retóricas (ver Tabla 4) como en el caso de la $M R 2$ citación de autores que aparece en varias de las secciones, esto puede reflejar un rasgo prototípico de la estructura retórica del género ponencia. Puede tomarse como un indicador de que uno de los propósitos comunicativos de este género se orienta hacia la legitimación de la investigación y puede funcionar discursivamente como una forma de persuasión dirigida a la audiencia.

\section{Tabla 4. Repetición de movidas en más de una sección}

Movida

Cita autores
Sección

I - II - IV - V 


\section{CONCLUSIONES}

Al final de esta investigación puede afirmarse que los objetivos planteados se han llevado a término, toda vez que se hizo una primera aproximación práctica que permitió esbozar la estructura retórica que conforma el género académico analizado, dando a conocer los propósitos comunicativos que subyacen al mismo.

Esta investigación permitió caracterizar la estructura retórica del género ponencia en cinco Secciones (I. Planteamiento de la investigación, II. Marco teórico y referencial, III. Diseño de la investigación, IV. Resultados y/o Discusión y V. Conclusiones), conformado por siete (7) movidas y trece (13) pasos. Sin embargo, la proyección investigativa apunta a indagar en un corpus mayor a fin de consolidar los resultados obtenidos.

Así mismo, se pudo identificar y explicar la función que cumple la movida MR2 citación de autores que más resaltó por la forma cíclica o reiterativa en que se presenta en este género. La función discursiva de $M R 2$ citación de autores, varía de acuerdo a su propósito comunicativo, pero finalmente va a incidir en la audiencia posicionando la investigación en un marco disciplinar de mayor alcance y relevancia. Los rasgos que caracterizan a este género el cual es presentado a un público especializado por medio de un discurso directo, le imprime mayor impacto ante la comunidad científica de que se trate.
Puede señalarse que al menos en la disciplina lingüística los autores se legitiman y posicionan sus investigaciones por medio de las $M R 2$ citación de autores.

Finalmente, los resultados obtenidos pueden ser considerados como un aporte para desarrollar la producción escrita de este tipo de texto académico, así como la competencia discursiva en estudiantes de pre y postgrado, lo que les permitirá su participación e incorporación en comunidades académicas especializadas.

\section{REFERENCIAS}

Briz, A. (coord.) (2008). Saber hablar. México: Instituto Cervantes, Aguilar

Carlino, P. (2005). ¿Por qué no se completan las tesis en los postgrados? Obstáculos percibidos por maestrandos en curso y magíster exitosos. Educere, (9)30, 415-420

Carlino, P. (2009). Escribir, leer y aprender en la universidad. Una introducción a la alfabetización académica. Buenos Aires: fce

Cubo de Severino, (2005). Escritura de formación en la universidad. En: Navarro (coordinador). Manual de escritura para carreras de humanidades. FILO: UBA

Duo de Brottier, O. (2007). La ponencia y el Resumen de Ponencia. En Los textos de la ciencia. Principales clases del discurso académico-científico. De Cubo de Severino. (2014).(Coordinadora). Córdoba, Argentina: Comunicarte editorial. Lengua y discurso 
Martín R., L. y Whittaker, R. (coord.) (1998). Poder-decir o el poder de los discursos. Madrid: Arrecife Producciones

Morales, O. (2010). Los Géneros Escritos de la Odontología Hispanoamericana. Estructura retórica y estrategias de atenuación en artículos de investigación, casos clinicos y artículos de revisión (tesis en línea). Recuperado de http:/ /www.tdx. cat/bitstream/ handle/10803/7577/tom.pdf?sequen $\mathrm{ce}=1$

Parodi, G. (2008) Géneros Académico y Géneros Profesionales: Accesos discursivo para saber y hacer. Valparaíso-Chile: Ediciones universitarias de Valparaíso. Pontificia

Parodi, G. (2010) La organización retórica del género Manual a través de cuatro disciplinas: ¿cómo se comunica y difunde la ciencia en diferentes contextos universitarios? Boletin de Lingüistica, XXII/33- 43-69. Recuperado de http://www.scielo.org.ve/scielo.php? pid=S0798-9709 201000010000 $3 \&$ script $=$ sciarttext

Sabaj, O., Toro, P. y Fuentes, M. (2011) Construcción de un modelo de movidas retóricas para el análisis de artículos de investigación en español. Onomazéin 24(2011/2), 245271.Recuperado de http://dialnet.unirioja.es/descarga/ar ticulo /3816395 . pdf

Santalla del Río, M. P. (2005). La elaboración de corpus lingüísticos. En
Cal, Núñez, y Palacios (eds.). Nuevas tecnologías en Lingüistica, Traducción y Enseñanza de lenguas. (pp. 45-66). Santiago: Universidad de Santiago de Compostela

Swales, J. M. (1990). Genre Analysis. English in academic and research settings. Australia: Cambridge

Scardamalia, M. y Bereiter, C. (1985). Develotment of dialectal processes in composition. En: Carlino, P. (2004). Escribir a través del currículum: tres modelos para hacerlo en la universidad. Lectura y vida. Revista Latinoamericana de lectura, 25(1), 16-27. Recuperado http://media.utp.edu.co/referenciasbibliograficas/uploads/ referencias/articulo/248-escribir-atravs-del-curriculum-tres-modelospara-hacerlo-en-la-universidadpdfjQyDB-articulo.pdf.

Van Dijk, T. (2009). Discurso y poder. Contribuciones a los estudios criticos del discurso. Barcelona: Editorial GEDISA

Venegas, R., Meza, P. y Martínez, J. (2013). Procedimientos discursivos en la atribución del conocimiento en Tesis de lingüística y Filosofía en los niveles académicos. RLA. Revista de Lingüistica Teórica y Aplicada Concepción (Chile), 51(1), 153-179. Recuperado de http://www.scielo.cl/scielo.php?pid= S0718-4883201300010000 8\&script=sci_arttext 